\title{
Total Duration of Negative Surplus for a Diffusion Surplus Process with Stochastic Return on Investments
}

\author{
Honglong You, Chuancun Yin \\ School of Mathematical Sciences, Qufu Normal University, Qufu, China \\ Email: ccyin@mail.qfnu.edu.cn, youhonglong815@163.com
}

Received August 31, 2012; revised September 30, 2012; accepted October 7, 2012

\begin{abstract}
In this paper, we consider a Brownian motion risk model with stochastic return on investments. Using the strong Markov property and exploiting the limitation idea, we derive the Laplace-Stieltjes Transform (LST) of the total duration of negative surplus. In addition, two examples are also present.
\end{abstract}

Keywords: Negative Surplus; Ruin Probability; Laplace-Stieltjes Transform

\section{Introduction}

Assume that the insurance business is described by the risk process

$$
U(t)=u+c t+\sigma B_{1}(t), \quad t \geq 0 .
$$

Here, $u$ is the initial capital; $c$ is the fixed rate of premium income; $\left\{B_{1}(x), t \geq 0\right\}$ is a standard Brownian motion; and $\sigma>0$ is a constant, representing the diffusion volatility.

Suppose that the insurer is allowed to invest in an asset or investment portfolio. Following Paulsen and Gjessing [1], we model the stochastic return as a Brownian motion with positive drift. Specifically, the return on the investment generating process is

$$
R(t)=r t+\delta B_{2}(t), \quad t \geq 0,
$$

where $r$ and $\delta$ are positive constants. In (1.2), $r$ is a fixed interest rate; $\left\{B_{2}(t), t \geq 0\right\}$ is another standard Brownian motion independent of $\left\{B_{1}(x), t \geq 0\right\}$, standing for the uncertainty associated with the return on investments at time $t$.

Let the risk process $\{X(t), t \geq 0\}$ denote the surplus of the insurer at time $t$ under this investments assumption. Thus, $X(t)$ associated with (1.1) and (1.2) is then the solution of the following linear stochastic integral equation:

$$
X(t)=U(t)+\int_{0}^{t} X(s) \mathrm{d} R(s) .
$$

By Paulsen [2] the solution of (1.3) is given by

$$
X(t)=R^{*}(t)\left(u+\int_{0}^{t}\left(R^{*}(s)\right)^{-1} \mathrm{~d} U(s)\right)
$$

where

$$
R^{*}(t)=\mathrm{e}^{\left(r-\frac{\delta^{2}}{2}\right) t+\delta B_{2}(t)} .
$$

Note that $\{X(t), t \geq 0\}$ is a homogeneous strong Markov process, see e.g. Paulsen and Gjessing [1].

The risk process (1.4) can be rewritten as

$$
\begin{aligned}
X(t)= & u+\int_{0}^{t} \delta X(s) \mathrm{d} B_{2}(s) \\
& +\int_{0}^{t} \sigma \mathrm{d} B_{1}(s)+\int_{0}^{t}(r X(s)+c) \mathrm{d} s .
\end{aligned}
$$

Because the quadratic variational processes of

$$
\int_{0}^{t} \delta X(s) \mathrm{d} B_{2}(s)+\int_{0}^{t} \sigma \mathrm{d} B_{1}(s)
$$

and

$$
\int_{0}^{t} \sqrt{\delta^{2} X^{2}(s)+\sigma^{2}} \mathrm{~d} B(s)
$$

are the same, where $\{B(t), t \geq 0\}$ is a standard Brownian motion, by Ikeda and Watanabe [3, p. 185] they have the same distribution. Thus, in distribution, we have

$$
\begin{aligned}
X(t)= & u+\int_{0}^{t} \sqrt{\delta^{2} X^{2}(s)+\sigma^{2}} \mathrm{~d} B(s) \\
& +\int_{0}^{t}(r X(s)+c) \mathrm{d} s .
\end{aligned}
$$

There are many papers concerning occupation times for different risk models. For example, for the classical surplus process with positive safety loading, Egdio dos Reis [4] derived the moment generating function of the total duration of the negative surplus by martingale methods, which was extended in Zhang and $\mathrm{Wu}$ [5] to the classical surplus process perturbed by diffusion. Chiu and Yin [6] derived explicit formula for the double Laplace-Stieltjes Transform (LST) of the occupation time in the exponential case for the compound Poisson model 
with a constant interest. He et al. [7] gived the LST of the total duration of negative surplus for the classical risk model with debit interest. More recently, Wang and $\mathrm{He}$ [8] considered the Brownian motion risk model with interest and derived the LST of total duration of negative surplus. In this paper, we consider a Brownian motion risk model with stochastic return on investments. We will use the limitation idea to obtain the LST of total duration of negative surplus.

The remainder of the paper is organized as follows. In Section 2, we give some preliminary results. In Section 3, by exploiting the limitation idea together with the results obtained in Section 2, we obtain the LST of the total duration of negative surplus. In the last section, we present two examples.

\section{Preliminary Results}

Given $u \in(-b, a)$, where $-\frac{c}{r} \leq-b$, define

$T_{a}=\inf \{t \geq 0, X(t)=a\}$ and if the set is empty $T_{a}=\infty$,

$T_{0}=\inf \{t \geq 0, X(t)=0\}$ and if the set is empty $T_{0}=\infty$,

$T_{-b}=\inf \{t \geq 0, X(t)=-b\}$ and if the set is empty $T_{-b}=\infty$,

$$
\chi(u,-b)=P\left(T_{-b}<\infty \mid X(0)=u\right)=P_{u}\left(T_{-b}<\infty\right) .
$$

Lemma 2.1 The risk process (1.5) has the strong Markov property: for any finite stopping time $T$ the regular conditional expectation of $X(T+t)$ given $F_{T}$ is $E[X(T+t) \mid X(T)]$, that is

$$
E\left[X(T+t) \mid F_{T}\right]=E[X(T+t) \mid X(T)], \quad t \geq 0, \text { a.s. }
$$

where $F_{T}$ is the information about the process up to time $T$, and the equality holds almost surely.

Lemma 2.2 For $\lambda>r$, the following ordinary differential equation

$$
\frac{\sigma^{2}+\delta^{2} x^{2}}{2} f^{\prime \prime}(x)+(c+r x) f^{\prime}(x)=\lambda f(x)
$$

has two independent solutions

$$
\varphi_{+}(x)=\int_{-\infty}^{x}(x-t)^{\rho+1} K(t) \mathrm{d} t
$$

and

$$
\varphi_{-}(x)=\int_{x}^{\infty}(t-x)^{\rho+1} K(t) \mathrm{d} t,
$$

where

$$
K(t)=\left(\sigma^{2}+\delta^{2} t^{2}\right)^{-\left(1+\frac{\beta}{2}\right)} \exp \left\{-\frac{2 c}{\delta \sigma} \arctan \left(\frac{\delta t}{\sigma}\right)\right\},
$$

$$
\begin{gathered}
\beta=\sqrt{\left(\frac{2 r}{\delta^{2}}-1\right)^{2}+8 \frac{\lambda}{\delta^{2}}}-1, \\
\rho=\frac{1}{2}\left(\sqrt{\left(\frac{2 r}{\delta^{2}}-1\right)^{2}+8 \frac{\lambda}{\delta^{2}}}-\left(1+\frac{2 r}{\delta^{2}}\right)\right) .
\end{gathered}
$$

Proof. From Example 2.2 of Paulsen and Gjessing [1], we get the result.

Lemma 2.3 For $\lambda>r>0,-\frac{c}{r}<u<a$ and $J=\left[-\frac{c}{r}, a\right], \quad \tau(J)=T_{a} \wedge T_{-\frac{c}{r}}$, define

$\Theta_{+}\left(a,-\frac{c}{r}, \lambda, u\right)=E_{u}\left[\mathrm{e}^{-\lambda \tau(J)} I_{\{X(\tau(J))=a\}}\right]$,

$$
\Theta_{-}\left(a,-\frac{c}{r}, \lambda, u\right)=E_{u}\left[\mathrm{e}^{-\lambda \tau(J)} I_{\left\{X(\tau(J))=\frac{-c}{r}\right\}}\right],
$$

then

$$
\begin{aligned}
& \Theta_{+}\left(a,-\frac{c}{r}, \lambda, u\right)=\frac{\varphi_{+}\left(\frac{-c}{r}\right) \varphi_{-}(u)-\varphi_{+}(u) \varphi_{-}\left(\frac{-c}{r}\right)}{\varphi_{+}\left(\frac{-c}{r}\right) \varphi_{-}(a)-\varphi_{+}(a) \varphi_{-}\left(\frac{-c}{r}\right)}, \\
& \Theta_{-}\left(a,-\frac{c}{r}, \lambda, u\right)=\frac{\varphi_{-}(a) \varphi_{+}(u)-\varphi_{-}(u) \varphi_{+}(a)}{\varphi_{-}(a) \varphi_{+}\left(\frac{-c}{r}\right)-\varphi_{-}\left(\frac{-c}{r}\right) \varphi_{+}(a)},
\end{aligned}
$$

where $\varphi_{+}$and $\varphi_{-}$are given by (2.2) and (2.3).

Proof. The result can be found in Chapter 16 of Breiman [9].

Lemma 2.4 For any $u \in(-b, a)$, then

$$
P_{u}\left(T_{-b}<T_{a}\right)=\frac{S(u)-S(a)}{S(-b)-S(a)},
$$

where $S(x)=\int_{0}^{x} \mathrm{e}^{-\int_{0}^{2} \frac{2 r s+2 c}{\delta^{2} s+\sigma^{2}} \mathrm{~d} s} \mathrm{~d} z$ is a solution of the equation

$$
\frac{\sigma^{2}+\delta^{2} x^{2}}{2} f^{\prime \prime}(x)+(c+r x) f^{\prime}(x)=0 .
$$

Proof. By Dynkin's formula,

$$
\begin{aligned}
& \left.E_{u}\left[S\left(X\left(t \wedge T_{-b} \wedge T_{a}\right)\right)\right]-\int_{0}^{t \wedge T_{-b} \wedge T_{a}} L S(X(s))\right) \mathrm{d} s \\
& =S(u),
\end{aligned}
$$

where $L$ is the generator of diffusion (1.5). It follows that

$$
\begin{aligned}
& L S(X(s))=\frac{\sigma^{2}+\delta^{2} X^{2}(s)}{2} S^{\prime \prime}(X(s)) \\
& +(c+r X(s)) S^{\prime}(X(s))=0 .
\end{aligned}
$$


Therefore

$$
E_{u}\left[S\left(X\left(t \wedge T_{-b} \wedge T_{a}\right)\right)\right]=S(u) .
$$

Since $T_{-b} \wedge T_{a}$ is finite, it takes values $T_{a}$ with probability $P_{u}\left(T_{a}<T_{-b}\right)$ and $T_{-b}$ with the complimentary probability. Letting $t \rightarrow \infty$, we can assert, by dominated convergence, that

$$
\begin{aligned}
& E_{u}\left[S\left(X\left(T_{-b} \wedge T_{a}\right)\right)\right] \\
& =E_{u}[S(X(0))]=S(u) .
\end{aligned}
$$

Expanding the expectation on the left, we have

$$
\begin{aligned}
& E_{u}\left[S(a) I_{\left\{T_{a}<T_{-b}\right\}}\right]+E_{u}\left[S(-b) I_{\left\{T_{-b}<T_{a}\right\}}\right] \\
& =S(u) .
\end{aligned}
$$

This, together with $P_{u}\left\{T_{a}<T_{-b}\right\}+P_{u}\left\{T_{-b}<T_{a}\right\}=1$, gives the result (2.4).

Lemma 2.5 For $u \geq 0$, the ruin probability for the risk model (1.5) is given by

$$
\Psi(u)=P_{u}\left(T_{0}<\infty\right)=\frac{\int_{u}^{\infty} h(z) \mathrm{d} z}{\int_{0}^{\infty} h(z) \mathrm{d} z} .
$$

The probability that the surplus process $\{X(t), t \geq 0\}$ hit the level $-b$ is given by

$$
\chi(u,-b)=P_{u}\left(T_{-b}<\infty\right)=\frac{\int_{u}^{\infty} h(z) \mathrm{d} z}{\int_{-b}^{\infty} h(z) \mathrm{d} z} .
$$

where

$$
h(z)=\mathrm{e}^{-\int_{0}^{z} \frac{2 r s+2 c}{\delta^{2} s+\sigma^{2}} \mathrm{ds}} .
$$

Proof. By Lemma 2.4, one can derive (2.5) and (2.6).

\section{Total Duration of Negative Surplus}

In this section, we will derive the main result of this paper. We assume that the risk process (1.5) does not attain the critical level $\frac{-c}{r}$. For convenience, we assume that the initial surplus $u$ is positive.

Let the total duration of negative surplus be

$$
\Pi=\int_{0}^{\infty} I_{\left\{\frac{-c}{r}<X(t)<0\right\}} \mathrm{d} t .
$$

For $0<\varepsilon<\frac{c}{r}$, define two sequences of stopping times of the process (1.5):

$$
\begin{aligned}
& \gamma_{1}=\inf \{t>0, X(t)=-\varepsilon\} \quad\left(\gamma_{1}=\infty\right. \text { if the set is empty), } \\
& \sigma_{1}=\inf \left\{t>\gamma_{1}, X(t)=0, \frac{-C}{r}<X(s)<0, \gamma_{1}<s<t\right\}
\end{aligned}
$$

( $\sigma_{1}=\infty$ if the set is empty),

in general, for $k=2,3, \cdots$, recursively define

$$
\gamma_{k}=\inf \left\{t>\sigma_{k-1}, X(t)=-\varepsilon\right\} \quad\left(\gamma_{k}=\infty\right. \text { if the set is }
$$
empty),

$$
\sigma_{k}=\inf \left\{t>\gamma_{k}, X(t)=0, \frac{-c}{r}<X(s)<0, \gamma_{k}<s<t\right\}
$$

( $\sigma_{k}=\infty$ if the set is empty).

Let $\Lambda_{k}=\sigma_{k}-\gamma_{k}, k=1,2,3, \cdots$. Given $\sigma_{k}<\infty$ for some $k \geq 1$, from the strong Markov property of the surplus process, we obtain that the periods

$\Lambda_{i}, i=1,2,3, \cdots, k$ are mutually independent and have a common distribution. Let $N$ denote the number of $\Lambda_{i}$.

Set $T^{*}=\sum_{i=1}^{N} \Lambda_{i}$. By the monotone convergence theorem, we have

$$
\lim _{\varepsilon \rightarrow 0_{+}} E_{u}\left(\mathrm{e}^{-\lambda T^{*}}\right)=E_{u}\left(\mathrm{e}^{-\lambda \Pi}\right) .
$$

First we give the expression for $E_{u}\left(\mathrm{e}^{-\lambda T^{*}}\right)$ in the following Theorem 3.1.

Theorem 3.1 For $u>0$ and $\lambda>r>0$, the LST of $T^{*}$ is given by

$$
\begin{aligned}
& E_{u}\left(\mathrm{e}^{-\lambda T^{*}}\right)=1-\chi(u,-\varepsilon) \\
& +\frac{\chi(u,-\varepsilon)(1-\chi(0,-\varepsilon)) \Theta_{+}\left(0,-\frac{c}{r}, \lambda,-\varepsilon\right)}{1-\chi(0,-\varepsilon) \Theta_{+}\left(0,-\frac{c}{r}, \lambda,-\varepsilon\right)},
\end{aligned}
$$

where $\Theta_{+}\left(0,-\frac{c}{r}, \lambda,-\varepsilon\right)$ and $\chi(u,-\varepsilon)$ are given by Lemmas 2.3 and 2.5.

Proof. From Lemma 2.1, we can get

$$
\begin{aligned}
E_{u}\left(\mathrm{e}^{-\lambda \Lambda_{k}}\right) & =E_{-\varepsilon}\left(\mathrm{e}^{-\lambda T_{0}} I_{\left\{T_{0}<T\right.}\right) \\
& \left.=\Theta_{-c / r}\right\} \\
& \left(0,-\frac{c}{r}, \lambda,-\varepsilon\right)
\end{aligned}
$$

and

$$
\begin{aligned}
E_{0}\left(\mathrm{e}^{-\lambda \Lambda_{k}}\right) & =E_{-\varepsilon}\left(\mathrm{e}^{-\lambda T_{0}} I_{\left\{T_{0}<T_{-c / r}\right\}}\right) \\
& =\Theta_{+}\left(0,-\frac{c}{r}, \lambda,-\varepsilon\right)
\end{aligned}
$$

From strong Markov property of the surplus process, we get 


$$
\begin{aligned}
& E_{u}\left(\mathrm{e}^{-\lambda T^{*}}\right)=P_{u}(N=0)+\sum_{k=1}^{\infty} E_{u}\left(\mathrm{e}^{-\lambda \sum_{i=1}^{k} \Lambda_{i}} I_{(N=k)}\right) \\
& =1-\chi(u,-\varepsilon) \\
& +\sum_{k=1}^{\infty} E_{u}\left(\mathrm{e}^{-\lambda \sum^{i} \Lambda_{\Lambda_{i}}} I_{\left\{\sigma_{1}<\infty, \sigma_{2}-\sigma_{1}<\infty, \cdots, \sigma_{k}-\sigma_{k-1}<\infty, X\left(\sigma_{k}+t\right)>-\varepsilon, t \geq 0\right\}}\right) \\
& =1-\chi(u,-\varepsilon) \\
& +(1-\chi(0,-\varepsilon)) \\
& \cdot \sum_{k=1}^{\infty} \chi(u,-\varepsilon) E_{u}\left(\mathrm{e}^{-\lambda \Lambda_{1}}\right)\left[\chi(0,-\varepsilon) E_{0}\left(\mathrm{e}^{-\lambda \Lambda_{1}}\right)\right]^{k-1} \\
& =1-\chi(u,-\varepsilon)+(1-\chi(0,-\varepsilon)) \frac{\chi(u,-\varepsilon) E_{u}\left(\mathrm{e}^{-\lambda \Lambda_{1}}\right)}{1-\chi(0,-\varepsilon) E_{0}\left(\mathrm{e}^{-\lambda \Lambda_{1}}\right)} .
\end{aligned}
$$

This, together with (3.3) and (3.4), gives (3.2).

Theorem 3.2 For $u>0$ and $\lambda>r>0$, the LST of total duration of negative surplus is given by

$$
\begin{aligned}
& E_{u}\left(\mathrm{e}^{-\lambda \Pi}\right)=1-\Psi(u) \\
& +\frac{\Psi(u) \Theta_{+}\left(0,-\frac{c}{r}, \lambda, 0\right)}{1+\Theta_{+}^{\prime}\left(0,-\frac{c}{r}, \lambda, 0\right) \int_{0}^{\infty} h(z) \mathrm{d} z},
\end{aligned}
$$

where $\Theta_{+}\left(0,-\frac{c}{r}, \lambda,-\varepsilon\right), \Psi(u)$ and $h(z)$ are given by Lemmas 2.3 and 2.5 .

Proof. It follows from (3.1) and (3.2) that

$$
\begin{aligned}
& E_{u}\left(\mathrm{e}^{-\lambda \Pi}\right) \\
= & \lim _{\varepsilon \rightarrow 0} E_{u}\left(\mathrm{e}^{-\lambda T^{*}}\right) \\
= & \lim _{\varepsilon \rightarrow 0}(1-\chi(u,-\varepsilon)) \\
& +\lim _{\varepsilon \rightarrow 0} \frac{\chi(u,-\varepsilon)(1-\chi(0,-\varepsilon)) \Theta_{+}\left(0,-\frac{c}{r}, \lambda,-\varepsilon\right)}{1-\chi(0,-\varepsilon) \Theta_{+}\left(0,-\frac{c}{r}, \lambda,-\varepsilon\right)} \\
= & 1-\Psi(u) \\
+ & \Psi(u) \lim _{\varepsilon \rightarrow 0} \frac{(1-\chi(0,-\varepsilon)) \Theta_{+}\left(0,-\frac{c}{r}, \lambda,-\varepsilon\right)}{1-\chi(0,-\varepsilon) \Theta_{+}\left(0,-\frac{c}{r}, \lambda,-\varepsilon\right)}
\end{aligned}
$$

From Lemma 2.5, it follows that

$$
\begin{aligned}
& \lim _{\varepsilon \rightarrow 0} \frac{(1-\chi(0,-\varepsilon)) \Theta_{+}\left(0,-\frac{c}{r}, \lambda,-\varepsilon\right)}{1-\chi(0,-\varepsilon) \Theta_{+}\left(0,-\frac{c}{r}, \lambda,-\varepsilon\right)} \\
& =\lim _{\varepsilon \rightarrow 0} \frac{\left(1-\frac{\int_{0}^{\infty} h(z) \mathrm{d} z}{\left.\int_{-\varepsilon}^{\infty} h(z) \mathrm{d} z\right)} \Theta_{+}\left(0,-\frac{c}{r}, \lambda,-\varepsilon\right)\right.}{1-\frac{\int_{0}^{\infty} h(z) \mathrm{d} z}{\int_{-\varepsilon}^{\infty} h(z) \mathrm{d} z} \Theta_{+}\left(0,-\frac{c}{r}, \lambda,-\varepsilon\right)} \\
& =\lim _{\varepsilon \rightarrow 0} \frac{\Theta_{+}\left(0,-\frac{c}{r}, \lambda,-\varepsilon\right) \int_{-\varepsilon}^{0} h(z) \mathrm{d} z}{\int_{-\varepsilon}^{\infty} h(z) \mathrm{d} z-\Theta_{+}\left(0,-\frac{c}{r}, \lambda,-\varepsilon\right) \int_{0}^{\infty} h(z) \mathrm{d} z} .
\end{aligned}
$$

By $\ell^{\prime}$ Hospital's rule, we get

$$
\begin{aligned}
\lim _{\varepsilon \rightarrow 0} \frac{\Theta_{+}\left(0,-\frac{c}{r}, \lambda,-\varepsilon\right) \int_{-\varepsilon}^{0} h(z) \mathrm{d} z}{\int_{-\varepsilon}^{\infty} h(z) \mathrm{d} z-\Theta_{+}\left(0,-\frac{c}{r}, \lambda,-\varepsilon\right) \int_{0}^{\infty} h(z) \mathrm{d} z} & =\lim _{\varepsilon \rightarrow 0} \frac{-\Theta_{+}^{\prime}\left(0,-\frac{c}{r}, \lambda,-\varepsilon\right) \int_{-\varepsilon}^{0} h(z) \mathrm{d} z+\Theta_{+}\left(0,-\frac{c}{r}, \lambda,-\varepsilon\right) h(-\varepsilon)}{h(-\varepsilon)+\Theta_{+}^{\prime}\left(0,-\frac{c}{r}, \lambda,-\varepsilon\right) \int_{0}^{\infty} h(z) \mathrm{d} z} \\
& =\frac{\Theta_{+}\left(0,-\frac{c}{r}, \lambda, 0\right)}{1+\Theta_{+}^{\prime}\left(0,-\frac{c}{r}, \lambda, 0\right) \int_{0}^{\infty} h(z) \mathrm{d} z} .
\end{aligned}
$$

This, together with (3.6) and (3.7), gives (3.5).

\section{Examples}

In this section we consider two examples.

Example 4.1. Letting $\delta=0$ in (1.5), we get the risk model

$$
X(t)=u+\int_{0}^{t}(c+r X(s)) \mathrm{d} s+\int_{0}^{t} \sigma \mathrm{d} B(s) .
$$

From Cai et al. [10], we know that the two independent solutions of the differential equation

$$
\frac{\sigma^{2}}{2} f^{\prime \prime}(x)+(r x+c) f^{\prime}(x)=\lambda f(x)
$$

are

$$
\begin{aligned}
& \varphi_{+}(x) \\
& =(c+r x) \exp \left\{\frac{-(c+r x)^{2}}{r \sigma^{2}}\right\} M\left(1+\frac{\lambda}{2 r}, \frac{3}{2}, \frac{(c+r x)^{2}}{r \sigma^{2}}\right)
\end{aligned}
$$


and

$$
\varphi_{-}(x)=\exp \left\{\frac{-(c+r x)^{2}}{r \sigma^{2}}\right\} U\left(\frac{1}{2}+\frac{\lambda}{2 r}, \frac{1}{2}, \frac{(c+r x)^{2}}{r \sigma^{2}}\right)
$$

where $M$ and $U$ are called the confluent hypergeometric functions of the first and second kind respectively. More detail on confluent hypergeometric functions can be found in Abramowitz and Stegun [11].

By Lemmas 2.3 and 2.5, we get

$$
\begin{aligned}
\Theta_{+}\left(0, \frac{-c}{r}, \lambda,-\varepsilon\right) & =E_{-\varepsilon}\left[\mathrm{e}^{-\lambda\left(T_{0} \wedge T_{-c / r}\right)} I_{\left\{T_{0}<T_{-c / r}\right\}}\right] \\
& =\frac{\varphi_{+}(-\varepsilon)}{\varphi_{+}(0)}, \\
\chi(u,-\varepsilon) & =\frac{\int_{u}^{\infty} h(z) \mathrm{d} z}{\int_{-\varepsilon}^{\infty} h(z) \mathrm{d} z}, \\
\Psi(u) & =\frac{\int_{u}^{\infty} h(z) \mathrm{d} z}{\int_{0}^{\infty} h(z) \mathrm{d} z},
\end{aligned}
$$

where

$$
h(z)=\mathrm{e}^{-\frac{\left(r z^{2}+2 c z\right)}{\sigma^{2}}} .
$$

According to Theorems 3.1 and 3.2, we get

$$
\begin{aligned}
& E_{u}\left(\mathrm{e}^{-\lambda T^{*}}\right)= 1-\chi(u,-\varepsilon) \\
&+\frac{\chi(u,-\varepsilon)(1-\chi(0,-\varepsilon)) \varphi_{+}(-\varepsilon)}{\varphi_{+}(0)-\chi(0,-\varepsilon) \varphi_{+}(-\varepsilon)}, \\
& E_{u}\left(\mathrm{e}^{-\lambda \Pi}\right)=1-\Psi(u)+\frac{\Psi(u) \varphi_{+}(0)}{\varphi_{+}(0)+\varphi_{+}^{\prime}(0) \int_{0}^{\infty} h(z) \mathrm{dz}},
\end{aligned}
$$

where $\varphi_{+}, \chi(u,-\varepsilon)$ and $\Psi(u)$ are given by (4.2), (4.5) and (4.6).

Remark 4.1 The results (4.7) and (4.8) coincide with the main results in Wang and $\mathrm{He}$ [7].

Example 4.2. Letting $\delta=0$ and $r=0$ in (1.5), we get the risk model

$$
X(t)=u+c t+\sigma B(t)
$$

It is easy to obtain that the two independent solutions of the ordinary differential equation

$$
\frac{\sigma^{2}}{2} f^{\prime \prime}(x)+c f^{\prime}(x)=\lambda f(x)
$$

are

$$
\varphi_{+}(x)=\mathrm{e}^{\left(-c+\sqrt{c^{2}+2 \sigma^{2} \lambda}\right) x}
$$

and

$$
\varphi_{-}(x)=\mathrm{e}^{\left(-c-\sqrt{c^{2}+2 \sigma^{2} \lambda}\right) x} .
$$

By Lemmas 2.3 and 2.5, we get

$$
\begin{aligned}
\Theta_{+}(0,-\infty, \lambda,-\varepsilon) & =E_{-\varepsilon}\left[\mathrm{e}^{-\lambda T_{0}} I_{\left\{T_{0}<\infty\right\}}\right] \\
& =\mathrm{e}^{\left(-c+\sqrt{c^{2}+2 \sigma^{2} \lambda}\right)(-\varepsilon)} \\
h(z) & =\mathrm{e}^{\frac{-2 c z}{\sigma^{2}}} \\
\Psi(u) & =\mathrm{e}^{\frac{-2 c}{\sigma^{2}} u}
\end{aligned}
$$

and

$$
\chi(u,-\varepsilon)=\mathrm{e}^{\frac{-2 c}{\sigma^{2}}(u+\varepsilon)} .
$$

According to Theorems 3.1 and 3.2, we have

$$
\begin{aligned}
E_{u}\left(\mathrm{e}^{-\lambda T^{*}}\right)= & 1-\mathrm{e}^{\frac{-2 c}{\sigma^{2}}(u+\varepsilon)} \\
+ & \frac{\mathrm{e}^{\frac{-2 c}{\sigma^{2}}(u+\varepsilon)}\left(1-\mathrm{e}^{\frac{-2 c}{\sigma^{2}} \varepsilon}\right) \mathrm{e}^{\varepsilon\left(c-\sqrt{c^{2}+2 \sigma^{2} \lambda}\right)}}{1-\mathrm{e}^{\frac{-2 c}{\sigma^{2}} \varepsilon} \mathrm{e}^{\varepsilon\left(c-\sqrt{c^{2}+2 \sigma^{2} \lambda}\right)}}
\end{aligned}
$$

and

$$
E_{u}\left(\mathrm{e}^{-\lambda \Pi}\right)=1-\mathrm{e}^{\frac{-2 c}{\sigma^{2}} u}+\frac{\mathrm{e}^{\frac{-2 c}{\sigma^{2}} u}}{1-\left(c-\sqrt{c^{2}+2 \sigma^{2} \lambda}\right) \frac{\sigma^{2}}{2 c}} .
$$

\section{Conclusion}

In this paper, we have studied the diffusion model incorporating stochastic return on investments. We find the LST of the total duration of negative surplus of this process. However, if the risk model (1.1) is extended to a compound Poisson surplus process perturbed by a diffusion, it is difficult to make out. We leave this problem for further research.

\section{REFERENCES}

[1] J. Paulsen and H. K. Gjessing, "Optimal Choice of Dividend Barriers for a Risk Process with Stochastic Return on Investments," Insurance: Mathematics and Economics, Vol. 20, No. 3, 1997, pp. 215-223. doi:10.1016/S0167-6687(97)00011-5

[2] J. Paulsen, "Risk Theory in a Stochastic Economic Environment," Stochastic Processes and Their Applications, Vol. 46, No. 2, 1993, pp. 327-361. doi:10.1016/0304-4149(93)90010-2

[3] N. Ikeda and S. Watanabe, "Stochastic Differential Equations and Diffusion Processes," North-Holland Publishing Company, Amsterdam, 1981. 
[4] A. D. Egdio dos Reis, "How Long Is the Surplus below Zero?" Insurance: Mathematics and Economics, Vol. 12, No. 1, 1993, pp. 23-38. doi:10.1016/0167-6687(93)90996-3

[5] C. S. Zhang and R. Wu, "Total Duration of Negative Surplus for the Compound Poisson Process That Is Perturbed by Diffusion," Insurance: Mathematics and Economics, Vol. 39, No. 3, 2002, pp. 517-532.

[6] S. N. Chiu and C. C. Yin, "On Occupation Times for a Risk Process with Reserve-Dependent Premium," Stochastic Models, Vol. 18, No. 2, 2001, pp. 245-255. doi:10.1081/STM-120004466

[7] J. M. He, R. Wu and H. Y. Zhang, "Total Duration of Negative Surplus for the Risk Model with Debit Interest," Statistics and Probability Letters, Vol. 79, No. 10, 2009, pp. 1320-1326. doi:10.1016/j.spl.2009.02.005

[8] W. Wang and J. M. He, "Total Duration of Negative Surplus for a Brownian Motion Risk Model with Interest," Acta Mathematica Sinica, 2012, (Submitted).

[9] L. Breiman, "Probability," Addison-Wesley, Reading, 1968.

[10] J. Cai, H. U. Gerber and H. L. Yang, “Optimal Dividends in an Ornstein-Uhlenbeck Type Model with Credit and Debit Interest," North American Actuarial Journal, Vol. 10, No. 2, 2006, pp. 94-119.

[11] M. Abramowitz and I. A. Stegun, "Handbook of Mathematical Functions: With Formulas, Graphs, and Mathematical Tables United States Department of Commerce," US Government Printing Office, Washington DC, 1972. 